\title{
High Pressure Diffraction on Single Crystals with Hot Neutrons at MLZ
}

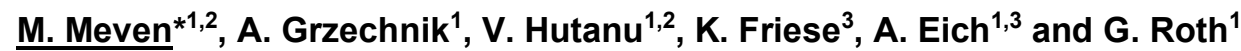 \\ ${ }^{1}$ Institute of Crystallography, RWTH Aachen University, 52056 Aachen, Germany \\ ${ }^{2} J u ̈ l i c h$ Centre for Neutron Science at Heinz Maier-Leibnitz Zentrum, Forschungszentrum Jülich GmbH, 85747 Garching, Germany \\ ${ }^{3}$ Jülich Centre for Neutron Science-2/Peter Grünberg-Institute-4, Forschungszentrum Jülich GmbH, 52425 Jülich, Germany \\ *martin.meven@frm2.tum.de
}

Due to their specific peculiarities neutrons are a very useful probe for structural studies on various hot topics related to physics, chemistry and mineralogy. The neutron single crystal diffractometer HEiDi at the Heinz Maier-Leibnitz Zentrum (MLZ) offers high flux, high resolution and large $q$ range, low absorption, and high sensitivity for light elements. These properties apply in a similar way to its polarized sister diffractometer POLI, which is optimized for detailed studies on magnetic structures.

In 2016 a project was launched in order to allow studies on tiny samples $<1 \mathrm{~mm}^{3}$ and to develop new pressure cells for HEiDi which can be combined with its existing low temperature equipment in order to study structural properties down to temperatures below $10 \mathrm{~K}$, e.g. $\mathrm{MnFe}_{4} \mathrm{Si}_{3}$ and related magnetocaloric compounds [1]. This work was supported by the Bundesministerium für Bildung und Forschung (BMBF) (grand no. 05K16PA3). As part of this project various neutron-optical components (Cu220-monochromator, solid state collimators, neutron guides) were developed and optimized in order to generate a sufficiently high flux density at the sample location at the wavelength $\lambda=0.87 \AA$. Very tiny single crystal samples (down to $<0.1 \mathrm{~mm}^{3}$ ) were successfully studied using various new diamond anvil cells (DAC) - developed by A. Grzechnik - up to several GPa, either with a panoramic pressure cell in combination with low temperatures [2] or in a transmission pressure cell, which allows simultaneous studies of the same sample using neutron, synchrotron as well as laboratory x-ray sources [3].

Recently, a follow up project has been launched (BMBF No. 05K19PA2) to focus on further improvements of the high-pressure capabilities on HEiDi and POLI and the development of optimized pressure cells for further instruments at the MLZ (POLI, DNS and MIRA), namely advanced clamp cells (see corresponding contribution by A. Eich).
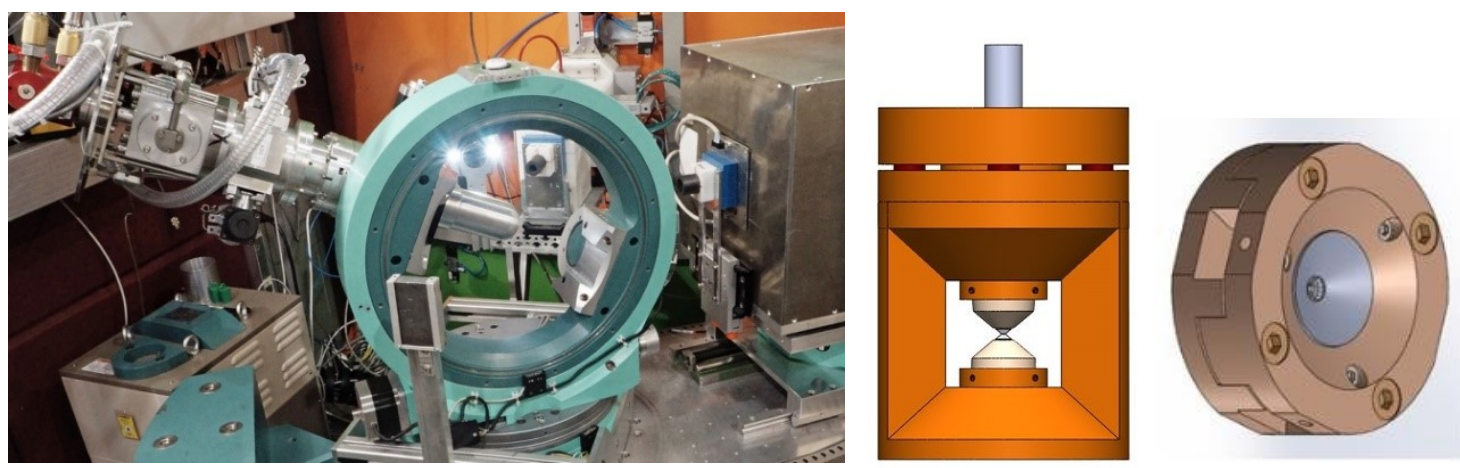

Figure 1: Single crystal diffractometer HEiDi (left), design studies of panoramic DAC (middle) and Yao-DAC (right).

[1] A. Grzechnik et al.; Single-Crystal Neutron Diffraction in Diamond Anvil Cells with Hot Neutrons; J. Appl. Cryst. 51, 351-356 (2018).

[2] A. Eich et al.; Magnetocaloric $\mathrm{Mn}_{5} \mathrm{Si}_{3}$ and $\mathrm{MnFe}_{4} \mathrm{Si}_{3}$ at variable pressure and temperature; Mater. Res. Express 6, 096118 (2019).

[3] A. Grzechnik et al.; Combined X-ray and neutron single-crystal diffraction in diamond anvil cells; J. Appl. Cryst. 53(1), 1 - 6 (2020).

Keywords: Neutron diffraction, High Pressure, diamond anvil cells, single crystal diffraction 